\title{
Slit beam shaping technique for femtosecond laser inscription of symmetric cladding waveguides
}

\author{
Pablo Roldán-Varona, Luis Rodríguez-Cobo, and José Miguel López-Higuera, Senior Member, IEEE
}

\begin{abstract}
In recent years, the Industry 4.0 paradigm has gained special relevance, focused on the integration of smart structures to increase the efficiency of processes. Photonics, with laser advanced manufacture and light-based sensors, can offer great possibilities in this field. Within advanced manufacturing, femtosecond lasers allow inscribing different optical structures. Waveguides are one of the most widely used structures in photonic sensors. This work proposes the design, simulation and manufacture of symmetric cladding waveguides in optical fibers using the slit beam shaping technique. Cladding waveguides characterized by their low losses $(0.21 \mathrm{~dB} / \mathrm{cm})$, quasi-step-index refractive index profile, and circular cross-section are used to couple evanescent light from the core of a single-mode fiber to each of the four cores of a multicore fiber.
\end{abstract}

Index Terms-Femtosecond laser, waveguide, optical fiber sensor, slit beam technique, multicore fiber, industry 4.0.

\section{INTRODUCTION}

$\mathbf{I}$ NDUSTRY 4.0, or 4th industrial revolution, involves the combination of existing advanced production techniques with smart technologies, with the aim of increasing the performance and efficiency of industry processes and services $[1,2]$. Among these new smart technologies, it can be highlighted advanced robotics, big data, internet of things (IoT) [3], maker culture [4], cognitive technologies [5], nanotechnology, or photonics, among many others.

Regarding the last mentioned technology, it seems clear that light science and technology is a key point in Industry 4.0 , as evidenced by the fact that it is one of the six key enabling technologies (KETs) that the European Commission considers fundamental to its relaunch and sustained economic growth [6]. Likewise, it is considered "essential" and "enabling technology" in the United States [7].

The use of light in Industry 4.0 can be understood from different points of view. First, the possibility of developing devices, subsystems and networks based on optical technologies allows advanced communications for the industry, an important characteristic for the interconnection of different smart architectures. Likewise, the light-based sensors stand

This work has been supported by the Ministerio de Ciencia, Innovación y Universidades and Agencia Estatal de Investigación (PID2019-107270RB$\mathrm{C} 21)$, the Ministerio de Educación, Cultura y Deporte of Spain (PhD grant FPU2018/02797), and FEDER funds. (Corresponding author: Pablo RoldánVarona)

P. Roldán-Varona (e-mail: pablo.roldan@ unican.es) and J.M. López Higuera (e-mail: miguel.lopezhiguera@unican.es) are with the Photonics Engineering Group, University of Cantabria, 39005, Santander, Spain; the CIBER-bbn, Instituto de Salud Carlos III, 28029, Madrid, Spain; and Instituto de Investigación Sanitaria Valdecilla (IDIVAL), 39011, Santander, Spain.

L. Rodríguez-Cobo (e-mail: luis.rodriguez@unican.es) is with the CIBERbbn, Instituto de Salud Carlos III, 28029, Madrid, Spain. out, which allow automating the collection of a large amount of physical, chemical, and/or biological information from the environment, being able to trigger concrete actions. Finally, with great industrial applicability, it is important to highlight advanced light-based manufacturing. Within this field, the micro-fabrication of 3D optical structures using femtosecond (fs) lasers is of notable importance [8,9].

In advanced manufacturing using fs lasers, waveguides are one of the most widely manufactured optical structures, due to their great applicability in the field of photonic sensors [9]. The possibility of optically interconnecting different sensor structures allows to manufacture more complete and efficient sensors, key points in Industry 4.0.

In this work, the Industry 4.0 paradigm is applied, when designing, simulating and manufacturing a light-based sensor inscribed by laser advanced manufacturing. In order to interrogate different optical structures in a very small space, a multicore fiber (MCF) composed of four cores is used. Individually, each core receives light through a cladding waveguide (CWG) that features an evanescent coupling with the core of a single-mode fiber (SMF). These waveguides, with a relatively circular cross-section, have been manufactured using the slit beam shaping technique $[10,11]$. To the best of the author's knowledge, this is the first time this method has been used to inscribe symmetric waveguides in optical fibers.

\section{FS LASER INSCRIPTION OF CLADDING WAVEGUIDES}

In recent years, although there are numerous studies in which waveguides have been manufactured using fs laser in bulk glasses [11-16], the manufacture of optical fiber waveguides appears significantly less in the literature. Optical fiber inscription presents important difficulties, associated with the size of the waveguides, as well as the coupling efficiency with the fiber core and other structures. However, the main problem is associated with the cylindrical geometry inherent to the fiber.

Considering optical fiber manufacturing, there are several ways to inscribe waveguides, as indicated in the review by Pallarés-Aldeiturriaga et al. [17]. There is a first case in which the cylindrical aberrations of the fiber are not corrected, giving rise to what is known as filamented waveguides [18]. To do this, high pulse energies are used, causing Type II modifications. The modified area of the focal volume presents a negative refractive index change (RIC), while the filament below presents a positive RIC $\left(\sim 10^{-2}\right)$, acting as a waveguide. However, the structure presents a clearly asymmetric cross-section, so its performance and mechanical robustness are very poor. 
In a second case, it is possible to overcome the cylinder lens effect of the fiber surface. To do this, there are basically two ways to proceed: use of adaptive optics (AO) [19] and/or index-matching oil in the fiber [20]. They can be used together. Adaptive optical elements allow spatial shaping of a laser beam in amplitude, polarization and phase, which offers great flexibility in aberration correction and beam shaping. Regarding the use of index-matching oil, it must be differentiated whether oil immersion is used [21, 22], or only the fiber is surrounded with oil and a coverslip is placed on top [23-25].

Since generally the waveguide width must be greater than the waist radius of the Gaussian beam focusing (focal volume) [26], it is necessary to use different approaches in order to achieve the appropriate size and shape. Considering the use of index-matching oil, the utilization of the multiscan technique is very common [23-25, 27]. This method consists in controlling the width (and shape) of the waveguide through multiple scans. Likewise, it is also possible to control the waveguide RIC by means of the appropriate overlap of the scans [25]. In this way, although the inscription time increases notably, a slight control of the cross-section of the waveguide is obtained without any increment in the optical complexity of the setup.

Through beam shaping, it is possible to control the shape of the waveguide cross-section without the requirement to perform multiple laser scans. There are different forms, which, although they cannot be strictly considered AO, allow to slightly correct the focal volume cross-section [26]. Among them, the slit beam shaping technique should be highlighted.

\section{A. Slit beam shaping technique}

The focal volume of a focused Gaussian beam is defined by the waist radius $\left(w_{0}\right)$ and the Rayleigh length $\left(z_{0}\right)$ :

$$
w_{0}=\frac{\lambda}{\pi \mathrm{NA}} \simeq \frac{\lambda}{\pi} \frac{f}{D}, \quad z_{0}=\frac{n \lambda}{\pi \mathrm{NA}^{2}},
$$

where NA is the numerical aperture of the objective lens, $f$ its focal length, $D$ the beam diameter at the lens input, $\lambda$ the laser wavelength, and $n$ the refractive index of the substrate. $w_{0}$ and $z_{0}$ can vary in each axis $(X, Y)$ if the NA is different in each axis, that is, if the diameter of the input beam $(D)$ is different in each axis. If the beam before the objective lens is elliptical, an asymmetric focal volume is achieved in the radial axis of the laser beam. One way to get an approach to an elliptical beam is by placing an adjustable slit before the objective lens.

Assuming that the longitudinal axis of the fiber is oriented in the direction of the $X$ axis, and that the cladding waveguides must be oriented in that axis, a circular cross-section in the $Y Z$ plane is required (Figure 1). Therefore, $w_{0 y}$ must be increased, that is, $D_{y}$ must be reduced at the input of the objective lens. By placing a slit oriented along the $X$ axis, where $a$ is the slit width (adjustable on the $Y$ axis), an elliptical beam can be assumed in the near field of the refractive element, that is, when the distance between the slit and the objective lens is within the Rayleigh range [28]:

$$
d_{\text {slit-lens }}<d_{R}=\frac{a^{2}}{\lambda} .
$$

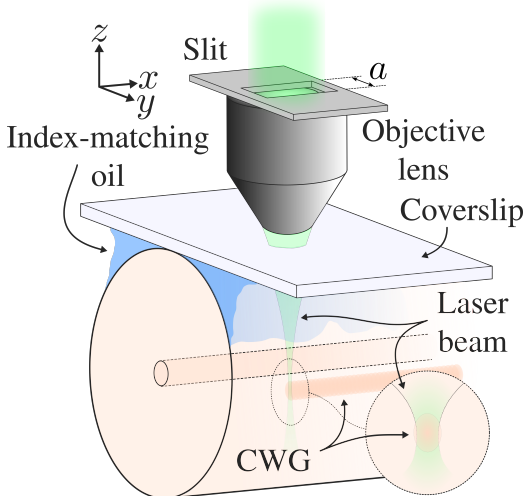

Fig. 1. CWG inscription scheme by means of slit beam shaping method, with index-matching oil.

The main advantage derived from the use of the slit beam shaping method for the manufacture of CWGs is the notably shorter inscription time compared to other techniques, such as multiscan. Likewise, a greater control of the waveguide crosssection is obtained, with a negligible increase in the optical complexity of the setup.

The slit beam shaping method was initially proposed by Ams et al. in 2005, for the inscription of circular waveguides in phosphate glass bulk [11]. This technique has also been employed on PMMA substrates [29]. In later years, in combination with active optical elements such as spatial light modulators (SLMs) [30, 31], or other techniques such as spatio-temporal focussing [32], the performance of the inscribed waveguides has been improved, always in bulk. In this work, to the best of the author's knowledge, the slit beam shaping technique is used for the first time for the inscription of cladding waveguides in optical fibers.

Additionally, it is important to note that this technique has not only been used for fs laser waveguide inscription. In 2020, Roldán et al. used this method to manufacture enhanced planeby-plane fiber Bragg gratings (FBGs), since they achieved planar RICs, completely covering the fiber core and thus improving the performance of gratings [10].

As a summary, Table I shows a comparison of some of the most relevant characteristics of the waveguide inscription techniques discussed in this section.

TABLE I. Outstanding characteristics of the different ways of inscribing waveguides with femtosecond laser. Filled circles indicate a high value in the parameter being evaluated. Empty

\begin{tabular}{|c|c|c|c|c|}
\hline & \multirow{2}{*}{$\begin{array}{l}\text { Direct-write } \\
\text { (filamented) }\end{array}$} & \multicolumn{2}{|c|}{ Index-matching oil } & \multirow{2}{*}{$\begin{array}{l}\text { Adaptive } \\
\text { optics }\end{array}$} \\
\hline & & Multiscan & $\begin{array}{c}\text { Slit beam } \\
\text { method }\end{array}$ & \\
\hline $\begin{array}{l}\text { Aberration } \\
\text { free }\end{array}$ & No & Yes & Yes & Yes \\
\hline $\begin{array}{l}\text { Inscription } \\
\text { time }\end{array}$ & - ० ० & $\bullet \bullet \bullet$ & - ० ० & - ० ० \\
\hline $\begin{array}{l}\text { Cross-section } \\
\text { control }\end{array}$ & $\circ \circ \circ$ & $\bullet \circ \circ$ & - ० ० & $\bullet \bullet \bullet$ \\
\hline $\begin{array}{c}\text { Light } \\
\text { transmission }\end{array}$ & - ० ० & $\bullet \bullet \circ$ & $\bullet \bullet \circ$ & $\bullet \bullet \bullet$ \\
\hline $\begin{array}{c}\text { Optical } \\
\text { complexity }\end{array}$ & $\circ \circ \circ$ & $\circ \circ \circ$ & $\bullet \circ \circ$ & $\bullet \bullet \bullet$ \\
\hline
\end{tabular}
circles indicate the opposite. 


\section{STRUCTURE DESIGN AND SIMULATIONS}

This work aims to show, for the first time, the ability to manufacture smooth and circular waveguides in optical fibers using the previously mentioned slit beam shaping technique. As proof of concept, the optical structure depicted in Figure 2 is designed, simulated and manufactured. It consists in coupling light from the SMF core to each of the MCF cores by means of evanescent wave coupling.

CWGs are made up of three clearly differentiated sections. A first straight section of length $L_{1}$, parallel to the SMF core and as close as possible to it, presents evanescent wave coupling. The fact of not inducing modifications on the core limits losses, allowing multiple optical structures to be placed along the fiber. The second section $\left(L_{2}\right)$ is an S-Bend that allows the light to be guided from the SMF core to the cladding, where through a last straight section $\left(L_{3}\right)$, it is coupled to the MCF core.

Previously detailed, by using the slit beam method it is possible to obtain a markedly circular waveguide cross-section [10]. Considering a $2.5 \mathrm{~mm}$ diameter $\left(1 / e^{2}\right)$ circular Gaussian beam, Figure 3 depicts the focal volume in the $Y Z$ plane, as well as the intensity curve in the $Z$ focal plane $(z=$ $62.5 \mu \mathrm{m} \rightarrow$ fiber center). The three cases presented are the following: without using a slit (Figure 3a), with $a=500 \mu \mathrm{m}$ slit (Figure 3b), as well as using a slit with $a=500 \mu \mathrm{m}$ and performing two scans with a $2.5 \mu \mathrm{m} Y$ offset (Figure 3c). The white circle corresponds to the circumference that delimits an SMF core, in order to obtain a relative view of the waveguide dimensions.

Looking at Figure 3b, it can be seen that the laser energy distribution at the focal point generates a more circular crosssection, but it seems insufficient in terms of the CWG diameter (compared to fiber core). There are two approaches to achieve a similar width to the core, and with a marked symmetry: decreasing the slit width $(a)$, or perform another scan with a slight $Y$ offset. The first approach is valid in theory, being achieved with $a \simeq 170 \mu \mathrm{m}$. However, in practice the energy that passes through the slit is so low that a significantly higher laser pulse energy would be required than that available in our setup. The second option (Figure 3c) consists of performing two scans with a slight $Y$ offset between them. In this way, it can be observed that a CWG width similar to the core and a circular symmetry in the cross-section are obtained.

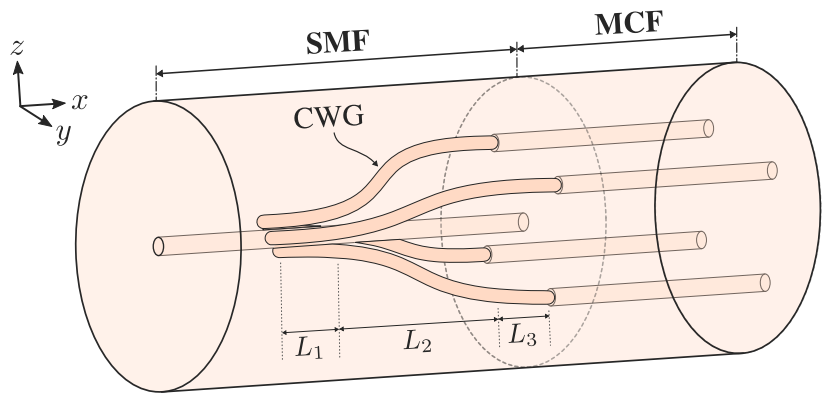

Fig. 2. Schematic view of the simultaed and manufactured optical structure. It consists of the evanescent coupling of light from the SMF core to each of the four cores of an MCF, through the use of symmetric cladding waveguides.
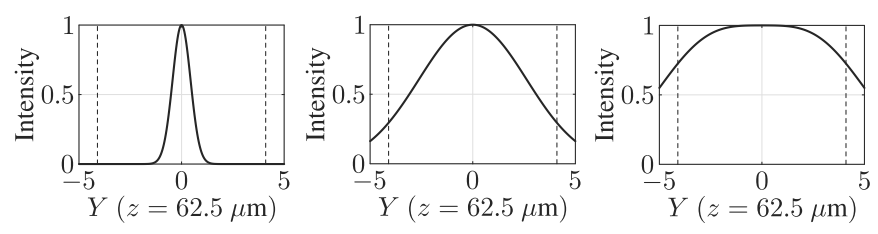

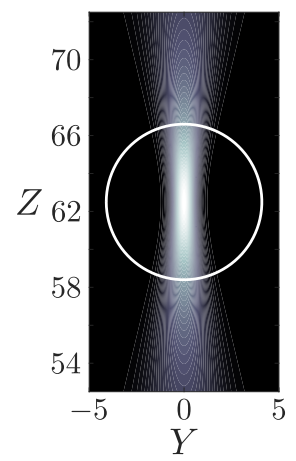

(a)

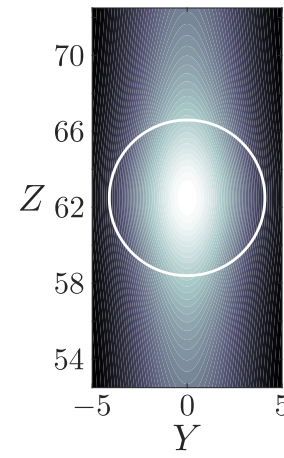

(b)

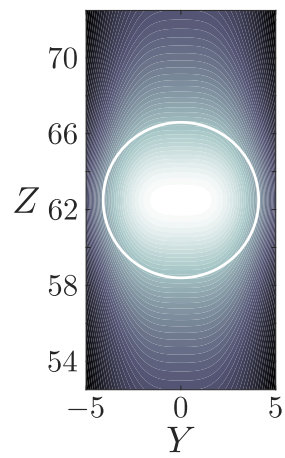

(c)
Fig. 3. $Y Z$ plane of the focal volume and intensity profile at $Z$ focus for focusing (a) without slit, (b) with a $a=500 \mu \mathrm{m}$ slit, (c) and with a $a=$ $500 \mu \mathrm{m}$ slit and performing two scans with a $2.5 \mu \mathrm{m} Y$ offset. A $f=8 \mathrm{~mm}$ focal length of the objective lens has been used, as well as refractive index adaptation (index-matching oil).

Additionally, considering the accumulated focal intensity of both scans, it is observed that it presents a flatter profile than in the case of a single scan. In this way, taking into account that the quantitative RIC is a function dependent on the pulse energy (in silica [9]), that is, on the energy density in the focal volume, the intensity profile offers an overview of the CWG refractive index profile. According to this, by performing two scans, a quasi-step-index CWG is obtained, compared to a profile that corresponds to a graded-index if a single scan is performed. This fact will be verified later by quantitative phase-contrast microscopy (QPM) measurements.

Next, simulations are carried out in order to establish the optimal lengths of sections $L_{1}, L_{2}$, and $L_{3}$ of the CWG. For this, the finite element beam propagation method (BPM) of RSoft software is used. It is defined the 2D structure generated by a longitudinal plane of the fiber of Figure 2 that contains the axial axes of the SMF core, as well as those of two cores of the MCF (separation between two MCF cores in that plane is $50 \mu \mathrm{m})$. The refractive index profile of CWGs is quasi-stepindex, and the coupling section is separated $1 \mu \mathrm{m}$ from the SMF core (transverse distance). The $L_{3}$ parameter is set to a value of $50 \mu \mathrm{m}$, with $L_{1}$ and $L_{2}$ being the parameters whose length is to be optimized.

Figure $4 \mathrm{a}$ depicts the normalized power of $\mathrm{LP}_{01}$ mode coupled in a CWG as a function of the length of the coupling section $L_{1}$. It can be seen that the optimal length is around $200 \mu \mathrm{m}$. Using this value in $L_{1}$, and in this case making a sweep in $L_{2}$, it is determined from Figure $4 \mathrm{~b}$ that the optimal $\mathrm{S}$-Bend length is around $1 \mathrm{~mm}$. Shorter lengths cause notable losses due to the abrupt change of light direction, and longer lengths infer a transition so smooth that back-coupling to the SMF core occurs. The simulation indicates that, with the proposed design, the $\mathrm{LP}_{01}$ power coupled in a CWG reaches $\simeq 36 \%$. Obviously, if the power has to be distributed equally, 


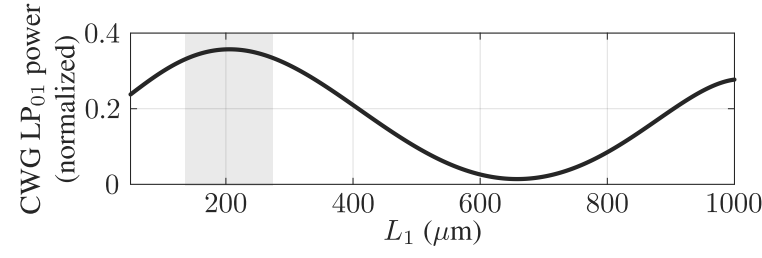

(a)

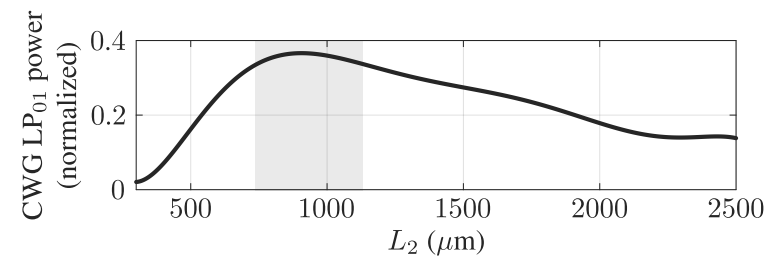

(b)

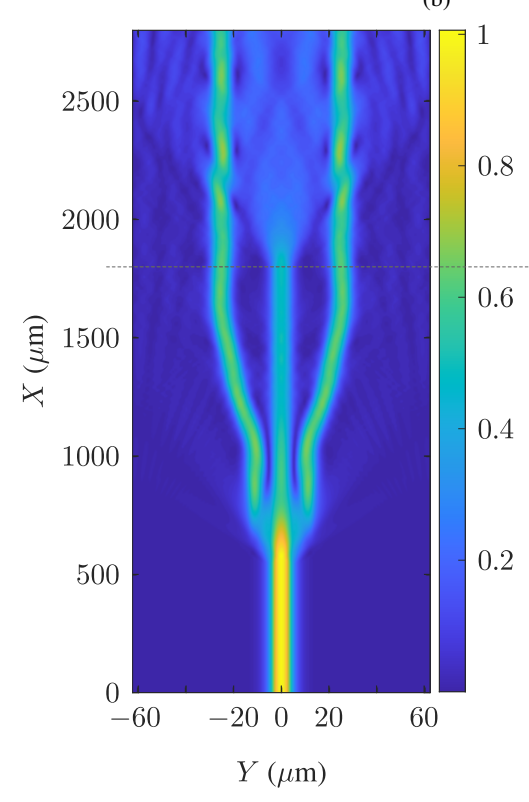

(c)
Fig. 4. Normalized $\mathrm{LP}_{01}$ power coupled to the CWG with $L_{3}=50 \mu \mathrm{m}$, and (a) varying $L_{1}$ (with $L_{2}=1 \mathrm{~mm}$ ), (b) or varying $L_{2}$ (with $L_{1}=200 \mu \mathrm{m}$ ). The shaded regions indicate the optimal design values. (c) (left) Filled 2D contour plot of the normalized $\mathrm{LP}_{01}$ power of the simulated structure, defined by the cut of the fiber indicated in Figure 2 by an $X Y$ plane that contains the longitudinal axes of the SMF core and of two MCF cores. (right) Evolution along $X$ axis of the normalized $\mathrm{LP}_{01}$ power in the SMF core, and in the combination of CWG and MCF core (only one). The shaded region indicates the evanescent wave coupling section.

each MCF core should receive $25 \%$. However, in practice, the losses presented by the manufactured CWGs as well as the non-ideality of the cross-section mean that each CWG will not couple $\simeq 36 \%$ of the SMF core power. Hence, the manufacture of the CWG is carried out with the optimal values obtained in simulation.

Figure $4 \mathrm{c}$ shows a filled 2D contour plot of the normalized $\mathrm{LP}_{01}$ power of the simulated structure with the chosen parameter values: $L_{1}=200 \mu \mathrm{m}, L_{2}=1 \mathrm{~mm}$, and $L_{3}=50 \mu \mathrm{m}$. Likewise, it is indicated the evolution along the $X$ axis of the $\mathrm{LP}_{01}$ power in the SMF core, as well as in one of the arms made up of CWG and MCF core. It is worth highlighting, in this last graph, the coupling region by evanescent wave.

\section{EXPERIMENTAL RESULTS AND DISCUSSION}

\section{A. Setup and inscription parameters}

For all following inscriptions, a commercial fiber laser chirped pulse amplifier (FLCPA) from CALMAR laser has been applied. It has a $1030 \mathrm{~nm}$ wavelength, and a $370 \mathrm{fs}$ pulse duration. The beam, with a diameter of $2.5 \mathrm{~mm}\left(1 / e^{2}\right)$, passes through a $3 \mathrm{~mm}$ iris diaphragm (to provide a clean wavefront) and, subsequently, through the slit oriented along the $X$ axis (adjustable on the $Y$ axis) [10]. The last part corresponds to the tight focussing of the laser beam through a NA $=0.42, \times 50$ Mitutoyo objective lens. The fiber sample is covered with an index-matching oil [20], and is placed on a nano-resolution $\mathrm{XYZ}$ motor stage from Aerotech.

From the knowledge given by previous studies, as well as by experimental measurements, it is inferred that the light transmission in direct-write waveguides improves when inscribing with circular polarization [33] (lower roughness and losses). Consequently, a $\lambda / 4$ plate is placed in the optical path of the laser beam. The complete setup is depicted in Figure 5.

Regarding the inscription parameters, the CWGs have been manufactured using a laser pulse energy $\left(E_{p}\right)$ of $0.52 \mu \mathrm{J}$ (prior to the slit), and a pulse repetition rate (PRR) of $120 \mathrm{kHz}$, with 1000 pulses $/ \mu \mathrm{m}$. The slit has a width $a=500 \mu \mathrm{m}$, and for each CWG two separate laser scans have been made with an offset of $2.5 \mu \mathrm{m}$ in the $Y$ axis.

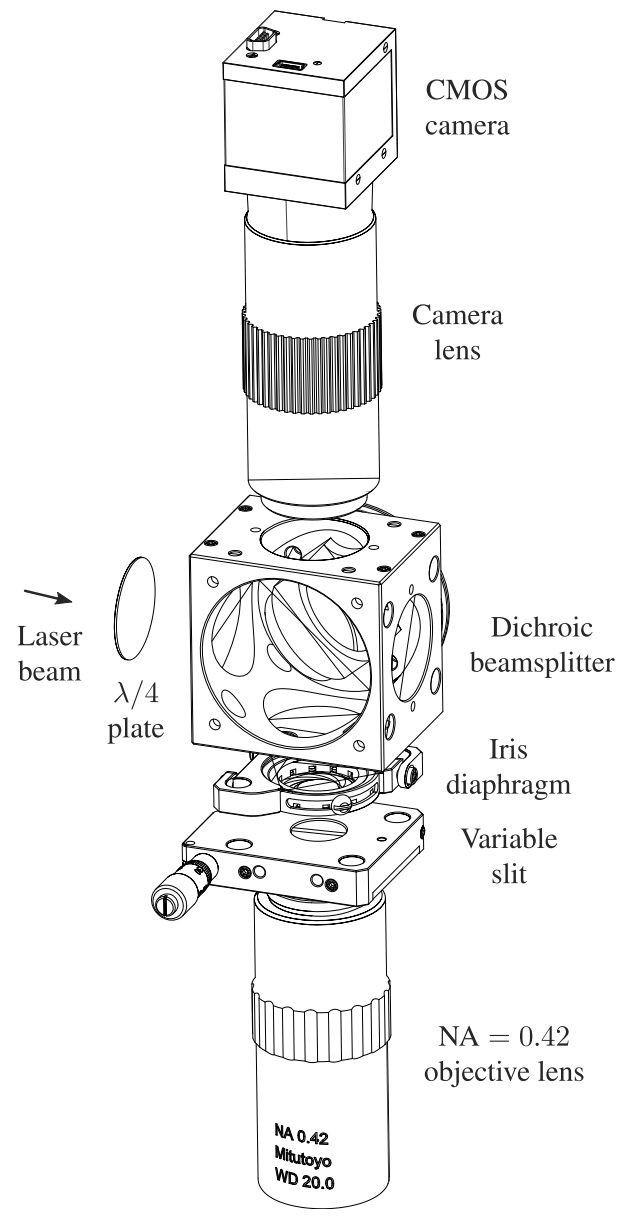

Fig. 5. Fs laser inscription setup used to manufacture cladding waveguides in optical fibers. Slit beam shaping technique is used. 


\section{B. CWG properties and cross-section}

The manufactured cladding waveguide has the previously defined dimensions $L_{1}, L_{2}$ and $L_{3}$, and a width of $8.1 \mu \mathrm{m}$, similar to the core width. The transverse separation with respect to the SMF core in the coupling section $\left(L_{1}\right)$ is $\sim 1 \mu \mathrm{m}$. This can be seen in Figure 6a. This closeness allows a relevant evanescent wave coupling, as can be deduced from the image in Figure $6 \mathrm{~b}$. The red light $(\lambda=633 \mathrm{~nm})$ introduced into the SMF is coupled to the guided modes in the waveguide, and the power contribution increases along the coupling section $\left(L_{1}\right)$ of the CWG. Red light scattering (indicative of waveguide losses) offers an explicit view of the amount of light coupled.

At this point, the waveguide losses have been estimated, for which image processing techniques have been used. Assuming that the CWG roughness remains practically constant throughout the waveguide, it is possible to indicate that the scattering and the guiding losses present a proportional relationship. In Figure $6 \mathrm{~b}$, the scattered red light is associated with losses in the cladding waveguide. The process carried out to estimate the losses has been as follows: (1) CWGs of different length $L_{1}$ are manufactured (only their coupling section). In this case, the manufactured waveguides have $L_{1}$ values of 100 , 200, 400, 600, 800, and $1000 \mu \mathrm{m}$. (2) Transmission power losses are obtained for each case. It has been used an optical

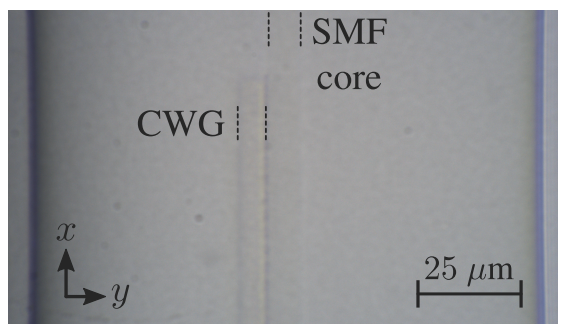

(a)

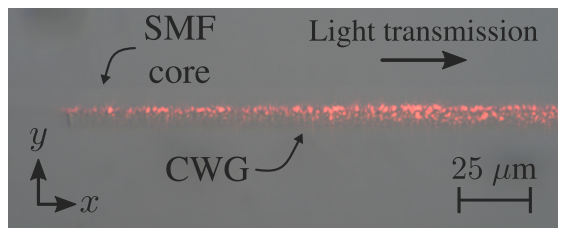

(b)

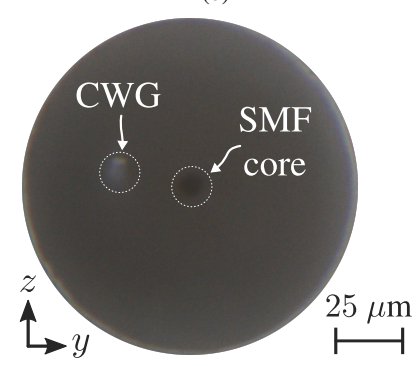

(c)

Fig. 6. (a) Microscope image $(\times 50)$ of the beginning of the CWG, in its coupling section. The transverse separation with respect to the SMF core is $1 \mu \mathrm{m}$. (b) Microscope image $(\times 20)$ of the coupling section of the CWG by introducing red light $(\lambda=633 \mathrm{~nm})$ at the distal end of the fiber. (c) Microscope image $(\times 20)$ of the end-face of the SMF fiber, with the CWG cross-section. spectrum analyzer (Anritsu MS9740A) with 30 pm resolution. (3) Images similar to the one depicted in Figure $6 \mathrm{~b}$ are obtained. Thus, there is a relationship between scattered light and guide losses (known) in each case. (4) Losses of each cladding waveguide (initially unknown) can be estimated based on its scattered light. The estimated loss value of manufactured CWGs is around $0.21 \mathrm{~dB} / \mathrm{cm}$.

The CWG cross-section is depicted in the end-face image of Figure $6 c$. It can be seen that its dimension is very similar to the SMF core, with an evident circular symmetry. In fact, its shape is identified with the focal volume obtained analytically in Figure 3c, made with the slit width and $Y$ offset (two scans).

Once the properties of the cladding waveguide have been specified, the structure outlined in Figure 2 is manufactured. Figure 7 depicts the result obtained. Figure 7 a shows the region corresponding to the splice between SMF and MCF. By focusing the image on the upper MCF cores (specifically on $\mathrm{MCF}$ core $_{1}$ ), it is possible to observe the waveguides corresponding to those cores. Ideally, the coupled power in each MCF core should be $25 \%$. However, as seen in Figure 7b, introducing red light at the distal end of the fiber (multimode behavior for that wavelength), three of them present a similar power coupling $\left(\mathrm{LP}_{31}\right.$ mode is guessed in them), while one seems to present a higher power level ( $\mathrm{LP}_{12}$ mode appears).

In any case, the CWGs manufactured are intended for use in 3rd window of optical communications (monomode behaviour). Specifically, a study of their behavior in C-band is carried out. For this, the HP $8168 \mathrm{~F}$ tunable laser (operating at $1550 \mathrm{~nm}$ with $0 \mathrm{dBm}$ power) and the Hamamatsu vidicon camera (in near field) are used, in order to quantify the power received in each core of the MCF, and thus establish the coupling ratio and the power loss. Figure 8 depicts the

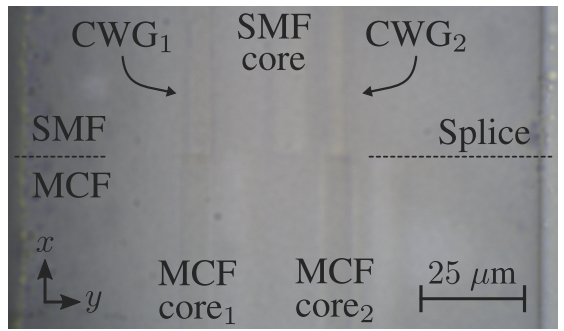

(a)

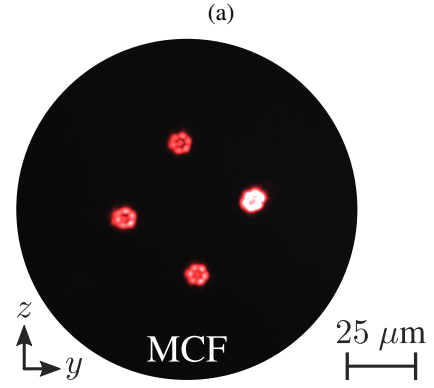

(b)

Fig. 7. (a) Microscope image $(\times 50)$ of the splice zone between SMF and MCF. By focusing the image on MCF core ${ }_{1}$, the associated CWG is observed. Likewise, the MCF core 2 and its $\mathrm{CWG}_{2}$ are also appreciated, despite being slightly out of focus. (b) Microscope image $(\times 20)$ of the end-face of the MCF fiber after manufacturing the complete structure of Figure 2, and introducing red light $(\lambda=633 \mathrm{~nm})$ at the distal end of the fiber. 
results obtained. The coupling factors of each core are $22.16 \%$, $23.68 \%, 29.83 \%$, and $24.33 \%$, respectively. Unlike the operation of these CWGs with visible wavelengths (Figure 7b), in this case only the fundamental mode of the guides is excited $\left(\mathrm{LP}_{01}\right)$. The losses of the complete optical structure are 2.78 $\mathrm{dB}$. Taking into account that the losses caused by the noncoupling of light from the SMF core are $1.36 \mathrm{~dB}$ (27\% of power is not coupled in the MCF cores), each CWG has absolute losses always lower than $0.4 \mathrm{~dB}$. Consequently, the results obtained are remarkably valid.

\section{QPM characterization}

Finally, it is important to characterize the RIC of the optical waveguide, or at least have a relative value with respect to the core. There are different ways to proceed. Quantitative phase microscopy (QPM) is one of them. This method allows to quantify the phase change that occurs when the light that illuminates the sample passes through an optically dense medium, such as optical fiber [34]. In this way, it is possible to extract a phase image from three intensity images, one being in focus $(\delta z=0)$ and the other two $\pm \delta z$ out of focus. It must be noted that the phase change $(\Delta \phi)$ and the RIC $(\Delta n)$ are related by the well-known relation:

$$
\Delta n=\frac{\lambda}{2 \pi \cdot z} \Delta \phi
$$

where $\lambda$ is the wavelength of the illumination source $(453 \mathrm{~nm})$, and $z$ is the inscription length (zone with different RI).

Figure 9 depicts the $Y$ phase change profile of an SMF with a cladding waveguide inscribed at $y=-30 \mu \mathrm{m}$, with the manufacturing parameters indicated above. It is observed that the phase change profiles of both structures (core and CWG) are similar. Therefore, their RI profile are similar as well. Likewise, it can be observed that the RIC value is slightly
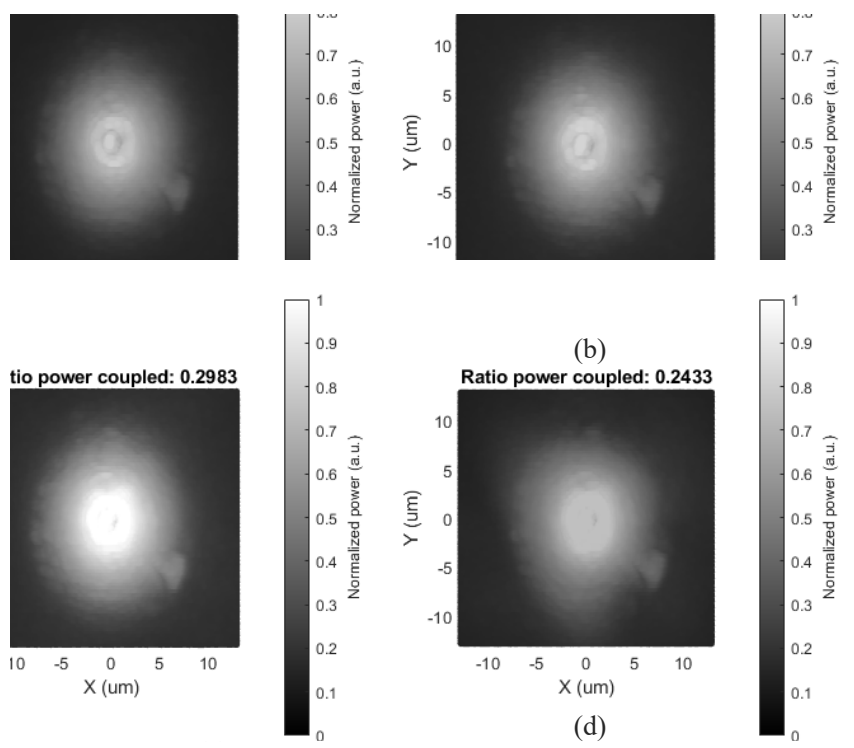

Fig. 8. Images corresponding to each of the four cores at the MCF end (origin of the axes has been defined relative to each core center). The spatial power patterns have been analyzed with a Hamamatsu vidicon camera. The power coupling factors in each core are (a) $22.16 \%$, (b) $23.68 \%$, (c) $29.83 \%$, and (d) $24.33 \%$.
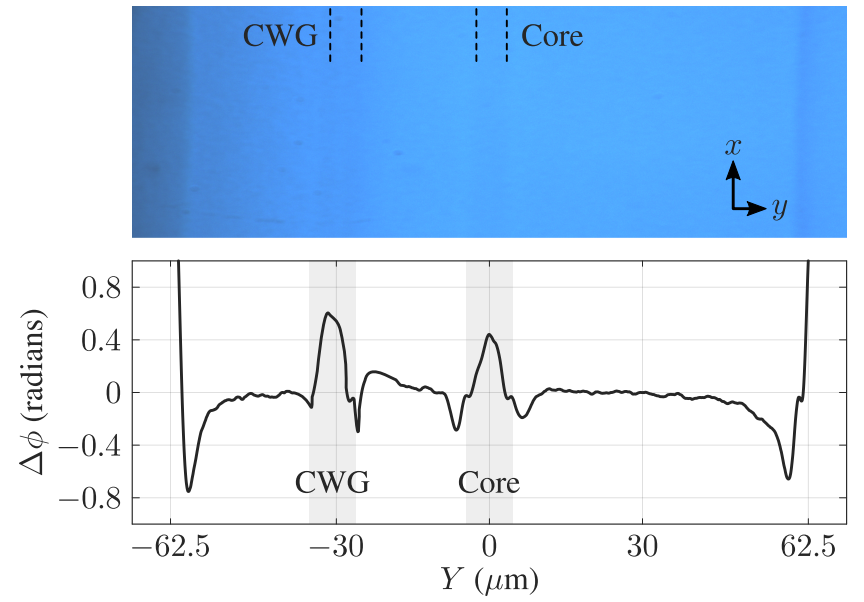

Fig. 9. Transverse phase profile measured with QPM for a cladding waveguide inscribed at $y=-30 \mu \mathrm{m}$.

higher in the CWG, but always in the order of magnitude of $10^{-3}$, suitable for transmission with low losses in waveguides.

\section{Sensor application}

Finally, as the last point of this work, its application as an optical sensor structure is briefly shown. For this, in addition to the scheme of Figure 2, four FBGs are inscribed, one in each core of the MCF, with a length of $1 \mathrm{~mm}$. The particularity lies in the fact that FBGs are manufactured, like CWGs, by means of the slit beam shaping technique. Consequently, the RIC induced by each pulse completely covers the core crosssection (Figure 10a), defining what is known as plane-by-plane (Pl-b-Pl) FBGs [10]. The inscription setup is, therefore, the one depicted in Figure 5.

Each FBG has been inscribed using a $1.09 \mu \mathrm{J}$ laser pulse energy (prior to slit), a slit width of $500 \mu \mathrm{m}$, a $300 \mathrm{~Hz}$ PRR, and a period such that the fourth order of the FBG originates

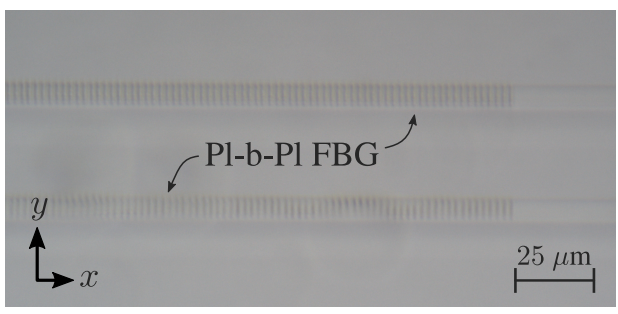

(a)

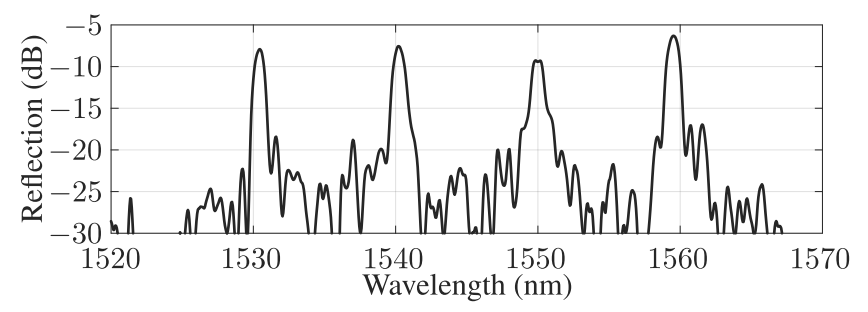

(b)

Fig. 10. (a) Microscope image of the multicore fiber, with a Pl-b-Pl FBG inscribed in each of the two focused cores. (b) Reflection spectrum resulting from the structure depicted in Figure 2, with a $1 \mathrm{~mm}$ long FBG inscribed in each core of the MCF. 
Bragg wavelengths at $1530,1540,1550$ and $1560 \mathrm{~nm}$, as seen in the reflection spectrum of Figure 10b.

In this way, as required by Industry 4.0, it allows integrating in a very small space $(1 \mathrm{~mm})$ four point sensor elements, capable of offering the value of different physical parameters, such as temperature, strain or bending; or even take advantage of the proximity to the surrounding medium to establish the sensing of different chemical quantities.

\section{CONCLUSION}

In this work, the slit beam shaping technique is used for the first time to inscribe waveguides in optical fibers using femtosecond lasers. It is presented the design, simulation and manufacture of a structure composed of four cladding waveguides that allows the evanescent light coupling from the core of a single-mode fiber to each of the cores of a multicore fiber. The cladding waveguides manufactured stand out for presenting a width of $8.1 \mu \mathrm{m}$ (single-mode behaviour in C-Band), a circular cross-section, as well as estimated losses of 0.21 $\mathrm{dB} / \mathrm{cm}$. Likewise, they have a slightly higher RIC than the core (in the same order of magnitude), with a quasi-step-index RI profile. The inscription parameters, as well as the design variables of the waveguides have been optimized through finite element simulations. Its great applicability for the advanced manufacture of light-based sensors in a reduced space provides great possibilities within Industry 4.0.

\section{ACKNOWLEDGMENT}

The authors would like to thank David Pallarés for the initial help on this topic.

\section{REFERENCES}

[1] H. Lasi, P. Fettke, H.-G. Kemper, T. Feld, and M. Hoffmann, "Industry 4.0," Business \& Information Systems Engineering, vol. 6, no. 4, pp. 239-242, jun 2014.

[2] L. D. Xu, E. L. Xu, and L. Li, "Industry 4.0: state of the art and future trends," International Journal of Production Research, vol. 56, no. 8, pp. 2941-2962, mar 2018.

[3] C. Zhang and Y. Chen, "A review of research relevant to the emerging industry trends: Industry 4.0, IoT, blockchain, and business analytics," Journal of Industrial Integration and Management, vol. 05, no. 01, pp. 165-180, feb 2020.

[4] T. Hamada, "Determinants of decision-makers' attitudes toward industry 4.0 adaptation," Social Sciences, vol. 8, no. 5, p. 140, may 2019.

[5] D. Romero, P. Bernus, O. Noran, J. Stahre, and Å. FastBerglund, "The operator 4.0: Human cyber-physical systems \& adaptive automation towards human-automation symbiosis work systems," in IFIP Advances in Information and Communication Technology. Springer International Publishing, 2016, pp. 677686.

[6] E. Commission, "Preparing for our future: Developing a common strategy for key enabling technologies in the EU," 2009.

[7] N. R. Council et al., Optics and photonics: Essential technologies for our nation. National Academies Press, 2013.

[8] R. R. Gattass and E. Mazur, "Femtosecond laser micromachining in transparent materials," Nature Photonics, vol. 2, no. 4, pp. 219-225, apr 2008.

[9] R. Osellame, G. Cerullo, and R. Ramponi, Eds., Femtosecond Laser Micromachining. Springer Berlin Heidelberg, 2012.

[10] P. Roldan-Varona, D. Pallares-Aldeiturriaga, L. RodriguezCobo, and J. M. Lopez-Higuera, "Slit beam shaping technique for femtosecond laser inscription of enhanced plane-by-plane FBGs," Journal of Lightwave Technology, vol. 38, no. 16, pp. 4526-4532, aug 2020.

[11] M. Ams, G. D. Marshall, D. J. Spence, and M. J. Withford, "Slit beam shaping method for femtosecond laser direct-write fabrication of symmetric waveguides in bulk glasses," Optics Express, vol. 13, no. 15, pp. 5676-5681, 2005.

[12] M. Will, S. Nolte, B. N. Chichkov, and A. Tünnermann, "Optical properties of waveguides fabricated in fused silica by femtosecond laser pulses," Applied Optics, vol. 41, no. 21, pp. 4360-4364, jul 2002.

[13] J. Siebenmorgen, T. Calmano, K. Petermann, and G. Huber, "Highly efficient Yb:YAG channel waveguide laser written with a femtosecond-laser," Optics Express, vol. 18, no. 15, pp. 16 035-16041, jul 2010.

[14] G. D. Valle, S. Taccheo, R. Osellame, A. Festa, G. Cerullo, and P. Laporta, " $1.5 \mu \mathrm{m}$ single longitudinal mode waveguide laser fabricated by femtosecond laser writing," Optics Express, vol. 15, no. 6, pp. 3190-3194, 2007.

[15] B. H. Babu, C. Lyu, T. Billotte, D. Pallares-Aldeiturriaga, B. Poumellec, J. M. Lopez-Higuera, X.-T. Hao, and M. Lancry, "Stress-induced optical waveguides written by an ultrafast laser in $\mathrm{Nd}^{3+}, \mathrm{Y}^{3+}$ co-doped $\mathrm{SrF}_{2}$ crystals," Applied Optics, vol. 58, no. 4, pp. 984-990, jan 2019.

[16] Y. Cheng, K. Sugioka, K. Midorikawa, M. Masuda, K. Toyoda, M. Kawachi, and K. Shihoyama, "Control of the cross-sectional shape of a hollow microchannel embedded in photostructurable glass by use of a femtosecond laser," Optics Letters, vol. 28, no. 1, pp. 55-57, jan 2003.

[17] D. Pallarés-Aldeiturriaga, P. Roldán-Varona, L. RodríguezCobo, and J. M. López-Higuera, "Optical fiber sensors by direct laser processing: A review," Sensors, vol. 20, no. 23, p. 6971, dec 2020.

[18] D. Pallares-Aldeiturriaga, L. Rodriguez-Cobo, A. Quintela, and J. M. Lopez-Higuera, "Curvature sensor based on in-fiber MachZehnder interferometer inscribed with femtosecond laser," Journal of Lightwave Technology, vol. 35, no. 21, pp. 4624-4628, nov 2017.

[19] P. S. Salter and M. J. Booth, "Adaptive optics in laser processing," Light: Science \& Applications, vol. 8, no. 1, pp. 1-16, nov 2019.

[20] Y. Lai, K. Zhou, L. Zhang, and I. Bennion, "Microchannels in conventional single-mode fibers," Optics Letters, vol. 31, no. 17, pp. 2559-2561, aug 2006.

[21] W. W. Li, W. P. Chen, D. N. Wang, Z. K. Wang, and B. Xu, "Fiber inline Mach-Zehnder interferometer based on femtosecond laser inscribed waveguides," Optics Letters, vol. 42, no. 21, pp. 4438-4441, oct 2017.

[22] Y. Zhang, C. Lin, C. Liao, K. Yang, Z. Li, and Y. Wang, "Femtosecond laser-inscribed fiber interface Mach-Zehnder interferometer for temperature-insensitive refractive index measurement," Optics Letters, vol. 43, no. 18, pp. 4421-4424, sep 2018.

[23] C. Waltermann, A. Doering, M. Köhring, M. Angelmahr, and W. Schade, "Cladding waveguide gratings in standard singlemode fiber for 3D shape sensing," Optics Letters, vol. 40, no. 13, pp. 3109-3112, jun 2015 .

[24] A. Theodosiou, A. Ioannou, and K. Kalli, "All-in-fiber cladding interferometric and Bragg grating components made via planeby-plane femtosecond laser inscription," Journal of Lightwave Technology, vol. 37, no. 18, pp. 4864-4871, sep 2019.

[25] P. Roldán-Varona, D. Pallarés-Aldeiturriaga, L. RodríguezCobo, and J. López-Higuera, "All-in-fiber multiscan MachZehnder interferometer assisted by core FBG for simultaneous multi-parameter sensing," Optics \& Laser Technology, vol. 132, p. 106459 , dec 2020.

[26] R. Osellame, G. Cerullo, and R. Ramponi, Eds., Femtosecond Laser Micromachining. Springer Berlin Heidelberg, 2012, ch. Controlling the Cross-section of Ultrafast Laser Inscribed 
Optical Waveguides, pp. 93-126.

[27] A. Theodosiou, A. Ioannou, M. Polis, A. Lacraz, C. Koutsides, and K. Kalli, "Femtosecond laser waveguide and FBG inscription in four-core optical fibre," in Micro-Structured and Specialty Optical Fibres IV, K. Kalli and A. Mendez, Eds. SPIE, apr 2016.

[28] C. S. Adams and I. G. Hughes, Optics f2f: From Fourier to Fresnel. Oxford University Press, dec 2018, ch. Many Waves I: Fresnel and Fraunhofer, pp. 71-90.

[29] S. Sowa, W. Watanabe, T. Tamaki, J. Nishii, and K. Itoh, "Symmetric waveguides in poly(methyl methacrylate) fabricated by femtosecond laser pulses," Optics Express, vol. 14, no. 1, pp. 291-297, 2006.

[30] J. Qi, P. Wang, Y. Liao, W. Chu, Z. Liu, Z. Wang, L. Qiao, and Y. Cheng, "Fabrication of polarization-independent singlemode waveguides in lithium niobate crystal with femtosecond laser pulses," Optical Materials Express, vol. 6, no. 8, pp. 25542559, jul 2016.

[31] P. S. Salter, A. Jesacher, J. B. Spring, B. J. Metcalf, N. ThomasPeter, R. D. Simmonds, N. K. Langford, I. A. Walmsley, and M. J. Booth, "Adaptive slit beam shaping for direct laser written waveguides," Optics Letters, vol. 37, no. 4, pp. 470-472, feb 2012.

[32] F. He, Y. Cheng, J. Lin, J. Ni, Z. Xu, K. Sugioka, and K. Midorikawa, "Independent control of aspect ratios in the axial and lateral cross sections of a focal spot for three-dimensional femtosecond laser micromachining," New Journal of Physics, vol. 13, no. 8, p. 083014, aug 2011.

[33] M. Ams, G. D. Marshall, and M. J. Withford, "Study of the influence of femtosecond laser polarisation on direct writing of waveguides," Optics Express, vol. 14, no. 26, pp. 13 158-13 163, 2006.

[34] C. J. Bellair, C. L. Curl, B. E. Allman, P. J. Harris, A. Roberts, L. M. D. Delbridge, and K. A. Nugent, "Quantitative phase amplitude microscopy IV: imaging thick specimens," Journal of Microscopy, vol. 214, no. 1, pp. 62-69, apr 2004.

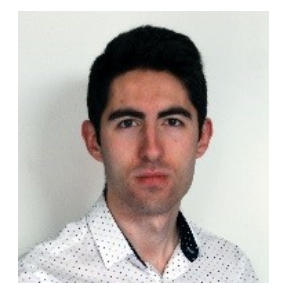

Pablo Roldán-Varona was born in Cantabria, Spain, in August 1995. He received the Bachelor's degree in Telecommunications Engineering from the University of Cantabria, Spain, in 2017; and the Master's degree in Telecommunications Engineering from the same university, in 2019. Currently, he is doing his $\mathrm{PhD}$ degree in the Photonics Engineering Group of the University of Cantabria. His research interests are in ultrafast laser processing and optical fiber sensors.

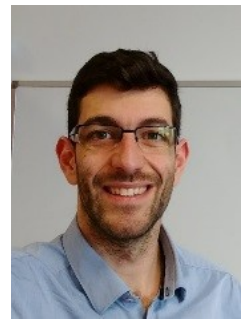

Luis Rodríguez-Cobo received his Ph.D. degree in Information and Communication Technologies (TICRM) from the University of Cantabria (Spain), in 2013. He has been awarded with the extraordinary $\mathrm{PhD}$. award for his works on optical sensors. After working as postdoctoral researcher at the Photonics Engineering Group (GIF), carrying out R\&D and project managing task, in 2017 he joined the CIBERBBN program of Institute of Health Carlos III as postdoctoral researcher to enhance its research in medical applications. His research is mainly focused on optical sensors, with particular attention to those based on optical fibers to be employed in many different scenarios.

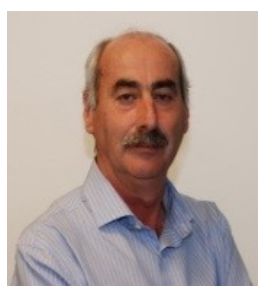

José Miguel López-Higuera (M'93-SM'98) was born in February 1954, in the village of Ramales de la Victoria, Cantabria, Spain. He obtained the Telecommunication Technical Engineering degree from the Universidad Laboral de Alcala de Henares, Madrid, Spain and the Telecommunication Engineering degree from the Universidad Politécnica de Madrid (UPM), Madrid, Spain. He received the $\mathrm{Ph} . \mathrm{D}$. degree in telecommunication engineering, with an extraordinary award, from UPM. He founded and is the Head of the Photonics Engineering Group of the TEISA Department in the University of Cantabria. Currently, he works in the development of Photonics Instrumentation, photonic/optical fibre sensor systems for civil engineering, electrical power, environmental and smart structures and for optical diagnostics for a wide range of applications. $\mathrm{He}$ has directed more than $50 \mathrm{R} \& \mathrm{D}$ projects and has written or co-written more than 400 publications in the form of books, chapters of books, papers and conferences, both national and international, and obtained 1ten patents. $\mathrm{He}$ is the editor and co-author of several books, including i) Optical Sensors (UC, 1998); ii) the Handbook of Photonic Sensing Technology (Wiley, 2002) and iii) he is the co-editor of the book Engineering a High-Tech Business: Entrepreneurial Experiences and Insights (SPIE-Press, 2008). Prof. LopezHiguera is Senior Member of the IEEE' and member of the IEE, SPIE and OSA. 